October 15, 2018

\title{
BPS Domain Walls and Vacuum Structure of $N=1$ Supergravity Coupled To A Chiral Multiplet
}

\author{
Bobby E. Gunara ${ }^{b}$ Freddy P. Zen ${ }^{b}$, and Arianto ${ }^{b, \sharp 1}$ \\ b Theoretical Physics Laboratory, \\ Theoretical High Energy Physics and Instrumentation Research Group, \\ Faculty of Mathematics and Natural Sciences, \\ Bandung Institute of Technology \\ Jl. Ganesha 10 Bandung 40132, Indonesia. \\ ${ }^{\sharp}$ Department of Physics, \\ Faculty of Mathematics and Natural Sciences, \\ Udayana University \\ Jl. Kampus Bukit Jimbaran-Kuta Denpasar 80361, Indonesia.
}

\begin{abstract}
We study BPS domain walls of $N=1$ supergravity coupled to a chiral multiplet and their Lorentz invariant vacuums which can be viewed as critical points of BPS equations and the scalar potential. Supersymmetry further implies that gradient flows of BPS equations controlled by a holomorphic superpotential and the Kähler geometry are unstable near local maximum of the scalar potential, whereas they are stable around local minimum and saddles of the scalar potential. However, the analysis using renormalization group flows shows that such gradient flows do not always exist particularly in infrared region.
\end{abstract}

\footnotetext{
${ }^{1}$ email: bobby@fi.itb.ac.id, fpzen@fi.itb.ac.id, feranie@upi.edu
} 


\section{Introduction}

Topological defects such as domain wall solutions of supergravity have acquired a large interest due to their duality with renormalization group $(R G)$ flows described by a beta function of field theory in the context of AdS/CFT correspondence [1]. In particular there has been a lot of study considering these solutions which preserve some fraction of supersymmetry in five dimensional supergravity theory, see, for example, in Ref. [2]. However, apart from this application our basic interest in domain walls is to investigate general properties of supersymmetric flows, namely gradient flows and RG flows, and supersymmetric Lorentz invariant vacuums of four dimensional $N=1$ supergravity coupled to a chiral multiplet. The walls preserve half of supersymmetry which are called BPS walls and were first discovered in Ref. [3] $]^{2}$.

The purpose of this paper is to present our study on BPS domain walls describing partial Lorentz invariant ground states of $N=1$ supergravity coupled to a chiral multiplet in four dimensions and properties of their $N=1$ critical points (equilibrium points) representing supersymmetric Lorentz invariant ground states, namely, Minkowskian and AdS ground states. The walls preserve half of supersymmetry and are described by BPS equations which can be seen as a set of two dimensional autonomous dynamical system ${ }^{3}$. Moreover, stability of gradient flows described by BPS equations is controlled by a holomorphic superpotential and the Kähler geometry.

Our first order analysis of BPS equations around critical points shows that in this model the gradient flows are unstable near local maximum of the scalar potential, whereas they are stable near saddles, local minimum, and degenerate critical points of the scalar potential. However, it turns out that such technique does not always work in general. First, the procedure fails when the flows become degenerate which occur near intrinsic degenerate critical points of the scalar potential ${ }^{4}$. Second, to get a complete picture one has to perform first order analysis on beta function. This analysis leads us to conclude that if parameters in the superpotential vary, in a region there is a possibility of having a zero eigenvalue in the analysis, then no critical points of the scalar potential exist in this region which follows that any gradient flow does not exist. For the case at hand, such situation occurs in infrared (low energy scale) region.

The organization of this paper is as follows. In section 2 we provide a quick review of four dimensional $N=1$ supergravity coupled to a chiral multiplet and also their supersymmetric solitonic solution BPS domain walls. In section 3 we consider properties of critical points describing supersymmetric ground states of BPS equations and scalar potential which are useful for our analysis in the next sections. Then we discuss the nature of BPS equation and beta function describing gradient and RG flows, respectively in section 4. Application of our analysis in several models is set in section 5. Finally we conclude our results in section 6 .

\footnotetext{
${ }^{2}$ BPS domain walls of four dimensional $N=1$ supergravity were extensively reviewed in Ref. [4]. We thank M. Cvetic for pointing out these references.

${ }^{3}$ The analysis using dynamical system has also been discussed for dilaton domain walls in Ref. [5].

${ }^{4}$ We distinguish the notion of intrinsic degenerate critical points with degenerate critical points of the scalar potential, see section 3 for detail.
} 


\section{BPS Domain Walls of $4 d N=1$ Supergravity Cou- pled to A Chiral Multiplet}

This section is devoted to give a short review of four dimensional $N=1$ supergravity coupled to a chiral multiplet and further discuss its solitonic solutions called domain walls which do not preserve fully Lorentz invariance. In particular, we only consider domain walls which inherit half of supersymmetry of the parental theory, i.e., the four dimensional $N=1$ supergravity. Such solution is called BPS domain wall ${ }^{5}$.

Let us first discuss the $N=1$ supergravity coupled to a chiral multiplet. This theory consists of a gravitational multiplet and a chiral multiplet. The gravitational multiplet contains a vierbein $e_{\mu}^{a}$ and a vector fermion $\psi_{\mu}$ where $a=0, \ldots, 3$ and $\mu=0, \ldots, 3$ are the flat and the curved indices, respectively. A chiral multiplet is composed of a complex scalar $z$ and a spin- $\frac{1}{2}$ fermion $\chi$.

The complete $N=1$ supergravity Lagrangian together with its supersymmetry transformation can be found, for example, in Ref. [7]. Here we collect the terms which are useful for our analysis. The bosonic part of the $N=1$ supergravity Lagrangian can be written as

$$
\mathcal{L}^{N=1}=-\frac{1}{2 M_{P}^{2}} R+g_{z \bar{z}} \partial_{\mu} z \partial^{\mu} \bar{z}-V(z, \bar{z}),
$$

where the complex scalars $(z, \bar{z})$ span a Hodge-Kähler manifold with metric $g_{z \bar{z}}=\partial_{z} \partial_{\bar{z}} K(z, \bar{z})$ and $K(z, \bar{z})$ is a real function called Kähler potential. The $N=1$ scalar potential $V(z, \bar{z})$ has the form

$$
V(z, \bar{z})=e^{K / M_{P}^{2}}\left(g^{z \bar{z}} \nabla_{z} W \bar{\nabla}_{\bar{z}} \bar{W}-\frac{3}{M_{P}^{2}} W \bar{W}\right),
$$

where $W$ is a holomorphic superpotential and $\nabla_{z} W \equiv(d W / d z)+\left(K_{z} / M_{P}^{2}\right) W$. The supersymmetry transformations up to three-fermion terms leaving invariant (2.1) are

$$
\begin{aligned}
\delta \psi_{1 \mu} & =\frac{1}{M_{P}} D_{\mu} \epsilon_{1}+\frac{\mathrm{i}}{2 M_{P}} e^{K / 2 M_{P}^{2}} W \gamma_{\mu} \epsilon^{1} \\
\delta \chi^{z} & =\mathrm{i} \partial_{\mu} z \gamma^{\mu} \epsilon^{1}+N^{z} \epsilon_{1} \\
\delta e_{\mu}^{a} & =-\mathrm{i} M_{P}\left(\bar{\psi}_{1 \mu} \gamma^{a} \epsilon^{1}+\bar{\psi}_{\mu}^{1} \gamma^{a} \epsilon_{1}\right) \\
\delta z & =\bar{\chi}^{z} \epsilon_{1}
\end{aligned}
$$

where $N^{z} \equiv e^{K / 2 M_{P}^{2}} g^{z \bar{z}} \bar{\nabla}_{\bar{z}} \bar{W}$ and $g^{z \bar{z}}=\left(g_{z \bar{z}}\right)^{-1}$. The coupling constant $M_{P}$ is the Planck mass that one regains $N=1$ global supersymmetric theory by setting $M_{P} \rightarrow+\infty$.

Now we will discuss the ground states which break partially Lorentz invariance, i.e., domain walls. First of all, one has to take the ansatz metric as

$$
d s^{2}=a^{2}(u) \eta_{\bar{\mu} \bar{\nu}} d x^{\bar{\mu}} d x^{\bar{\nu}}-d u^{2},
$$

\footnotetext{
${ }^{5}$ In this section some results have been discussed in Ref. [6] in the context of stringy landscape. We thank K. Yamamoto for noticing us this paper.
} 
where $\bar{\mu}, \bar{\nu}=0,1,2$ and $a(u)$ is the warped factor. Writing the supersymmetry transformation (2.3) and setting $\psi_{1 \mu}=\chi^{z}=0$ on the background (2.4), this leads

$$
\begin{aligned}
\delta \psi_{1 u} & =\frac{1}{M_{P}} D_{u} \epsilon_{1}+\frac{\mathrm{i}}{2 M_{P}} e^{K / 2 M_{P}^{2}} W \gamma_{u} \epsilon^{1}, \\
\delta \psi_{1 \bar{\mu}} & =\frac{1}{M_{P}} \partial_{\bar{\mu}} \epsilon_{1}+\frac{1}{2 M_{P}} \gamma_{\bar{\mu}}\left(-\frac{a^{\prime}}{a} \gamma_{3} \epsilon_{1}+\mathrm{i} e^{K / 2 M_{P}^{2}} W \epsilon^{1}\right)+\ldots \\
\delta \chi^{z} & =\mathrm{i} \partial_{\mu} z \gamma^{\mu} \epsilon^{1}+N^{z} \epsilon_{1},
\end{aligned}
$$

where $a^{\prime} \equiv d a / d u$ and the dots mean that these term can be neglected in our analysis. Furthermore, in order to have residual supersymmetry on the ground states, the equations in Eq. (2.5) have to be vanished. For the sake of simplicity, we assign that $\epsilon_{1}$ and $z$ are only $u$ dependent. Thus, the first equation in Eq. (2.5) shows that $\epsilon_{1}$ depends only on $u$, while the second equation gives a projection equation

$$
\frac{a^{\prime}}{a} \gamma_{3} \epsilon_{1}=\mathrm{i} e^{K / 2 M_{P}^{2}} W(z) \epsilon^{1}
$$

which leads to

$$
\frac{a^{\prime}}{a}= \pm e^{K / 2 M_{P}^{2}}|W(z)|
$$

The third equation in Eq. (2.5) becomes

$$
\begin{aligned}
& z^{\prime}=\mp 2 g^{z \bar{z}} \bar{\partial}_{\bar{z}} \mathcal{W}(z, \bar{z}), \\
& \bar{z}^{\prime}=\mp 2 g^{z \bar{z}} \partial_{z} \mathcal{W}(z, \bar{z}),
\end{aligned}
$$

where we have introduced a real function $\mathcal{W}(z, \bar{z}) \equiv e^{K / 2 M_{P}^{2}}|W(z)|$. The gradient flow equations (2.8) are called BPS equations. Note that using Eq. (2.8) one shows that the function (2.7) is monotonically decreasing and related to the monotonicity of the cfunction in the holographic correspondence [8]. Furthermore, from conformal field theory (CFT) point of view the relevant supersymmetric flows for all scalar fields can also be described by a beta function defined as

$$
\beta \equiv a \frac{d z}{d a}=-2 g^{z \bar{z}} \frac{\bar{\partial}_{\bar{z}} \mathcal{W}}{\mathcal{W}}
$$

after using Eqs. (2.7) and (2.8), together with its complex conjugate. In this picture the scalars can be viewed as coupling constants and the warp factor $a$ is playing the role of an energy scale $[8,9]$.

Now the potential (2.2) can be cast into the form

$$
V(z, \bar{z})=4 g^{z \bar{z}} \partial_{z} \mathcal{W} \bar{\partial}_{\bar{z}} \mathcal{W}-\frac{3}{M_{P}^{2}} \mathcal{W}^{2}
$$

and furthermore its first derivative with respect to $(z, \bar{z})$ is given by

$$
\begin{aligned}
& \frac{\partial V}{\partial z}=4 g^{z \bar{z}} \nabla_{z} \partial_{z} \mathcal{W} \bar{\partial}_{\bar{z}} \mathcal{W}+4 g^{z \bar{z}} \partial_{z} \mathcal{W} \partial_{z} \bar{\partial}_{\bar{z}} \mathcal{W}-\frac{6}{M_{P}^{2}} \mathcal{W} \partial_{z} \mathcal{W} \\
& \frac{\partial V}{\partial \bar{z}}=4 g^{z \bar{z}} \bar{\nabla}_{\bar{z}} \bar{\partial}_{\bar{z}} \mathcal{W} \partial_{z} \mathcal{W}+4 g^{z \bar{z}} \bar{\partial}_{\bar{z}} \mathcal{W} \bar{\partial}_{\bar{z}} \partial_{z} \mathcal{W}-\frac{6}{M_{P}^{2}} \mathcal{W} \bar{\partial}_{\bar{z}} \mathcal{W}
\end{aligned}
$$


where $\nabla_{z} \partial_{z} \mathcal{W}=\partial_{z}^{2} \mathcal{W}-\Gamma_{z z}^{z} \partial_{z} \mathcal{W}$

We give now our attention to the gradient flow equation (2.8) and the first derivative of the scalar potential (2.11). Critical points of Eq. (2.8) are related to the following condition

$$
\partial_{z} \mathcal{W}(z, \bar{z})=\bar{\partial}_{\bar{z}} \mathcal{W}(z, \bar{z})=0
$$

which implies that

$$
\frac{\partial V}{\partial z}=\frac{\partial V}{\partial \bar{z}}=0
$$

This means that the critical points of $\mathcal{W}(z, \bar{z})$ are somehow related to the critical points of the $N=1$ scalar potential $V(z, \bar{z})$. Moreover, in the view of Eq. (2.9), these points are in ultraviolet (UV) region if $a \rightarrow \infty$ and in infrared (IR) region if $a \rightarrow 0$. Thus, the RG flow interpolates between UV and IR critical points. These will be discussed in section 4 .

\section{Critical Points of $\mathcal{W}(z, \bar{z})$ and $V(z, \bar{z})$}

In this section our discussion will be focused on general properties of critical points of the real function $\mathcal{W}(z, \bar{z})$ and the $N=1$ scalar potential $V(z, \bar{z})$ describing supersymmetric Lorentz invariant vacuums ${ }^{6}$. General theory of critical points of surfaces can be found in, for example, Eq. [10] and Appendix B.

First of all we discuss the critical points of $\mathcal{W}(z, \bar{z})$. It is straightforward to write down the eigenvalues and the determinant of the Hessian matrix of $\mathcal{W}(z, \bar{z})$ evaluated at the critical point $p_{0} \equiv\left(z_{0}, \bar{z}_{0}\right)$,

$$
\begin{aligned}
\lambda_{1,2}^{\mathcal{W}} & =\frac{g_{z \bar{z}}\left(p_{0}\right)}{M_{P}^{2}} \mathcal{W}\left(p_{0}\right) \pm 2\left|\partial_{z}^{2} \mathcal{W}\left(p_{0}\right)\right| \\
\operatorname{det} H_{\mathcal{W}} & =\frac{g_{z \bar{z}}^{2}\left(p_{0}\right)}{M_{P}^{4}} \mathcal{W}^{2}\left(p_{0}\right)-4\left|\partial_{z}^{2} \mathcal{W}\left(p_{0}\right)\right|^{2}
\end{aligned}
$$

where

$$
\partial_{z}^{2} \mathcal{W}\left(p_{0}\right)=\frac{e^{K\left(p_{0}\right) / M_{P}^{2}} \bar{W}\left(\bar{z}_{0}\right)}{2 \mathcal{W}\left(p_{0}\right)}\left(\frac{d^{2} W}{d z^{2}}\left(z_{0}\right)+\frac{K_{z z}\left(p_{0}\right)}{M_{P}^{2}} W\left(z_{0}\right)+\frac{K_{z}\left(p_{0}\right)}{M_{P}^{2}} \frac{d W}{d z}\left(z_{0}\right)\right) .
$$

Local minimum point occurs if

$$
\left|\partial_{z}^{2} \mathcal{W}\left(p_{0}\right)\right|<\frac{1}{2 M_{P}^{2}} g_{z \bar{z}}\left(p_{0}\right) \mathcal{W}\left(p_{0}\right)
$$

while saddle point requires

$$
\left|\partial_{z}^{2} \mathcal{W}\left(p_{0}\right)\right|>\frac{1}{2 M_{P}^{2}} g_{z \bar{z}}\left(p_{0}\right) \mathcal{W}\left(p_{0}\right)
$$

Moreover, critical point becomes degenerate if

$$
\left|\partial_{z}^{2} \mathcal{W}\left(p_{0}\right)\right|=\frac{1}{2 M_{P}^{2}} g_{z \bar{z}}\left(p_{0}\right) \mathcal{W}\left(p_{0}\right)
$$

\footnotetext{
${ }^{6}$ In the paper we assume $\mathcal{W}(z, \bar{z})$ and $V(z, \bar{z})$ to be $C^{\infty}$-function.
} 
and in general there is no local maximum point for the model.

Now we investigate critical points of the scalar potential $V(z, \bar{z})$ and its relation to the critical points of $\mathcal{W}(z, \bar{z})$. The eigenvalues and the determinant of the Hessian matrix of $V(z, \bar{z})$ evaluated at the critical point $p_{0}$ can be expressed as follows,

$$
\begin{aligned}
\lambda_{1,2}^{V}= & -2\left(g^{z \bar{z}}\left(p_{0}\right) \operatorname{det} H_{\mathcal{W}}+\frac{g_{z \bar{z}}\left(p_{0}\right)}{M_{P}^{4}} \mathcal{W}^{2}\left(p_{0}\right)\right) \\
& \pm 2 \frac{\mathcal{W}\left(p_{0}\right)}{M_{P}^{2}}\left[\frac{g_{z \bar{z}}^{2}\left(p_{0}\right)}{M_{P}^{4}} \mathcal{W}^{2}\left(p_{0}\right)-\operatorname{det} H_{\mathcal{W}}\right]^{\frac{1}{2}}, \\
\operatorname{det} H_{V}= & 4 \operatorname{det} H_{\mathcal{W}}\left[g_{z \bar{z}}^{-2}\left(p_{0}\right) \operatorname{det} H_{\mathcal{W}}+\frac{3 \mathcal{W}^{2}\left(p_{0}\right)}{M_{P}^{4}}\right] .
\end{aligned}
$$

We find that local minimum of the scalar potential $V(z, \bar{z})$ has to be fulfilled

$$
\left|\partial_{z}^{2} \mathcal{W}\left(p_{0}\right)\right|>\frac{g_{z \bar{z}}\left(p_{0}\right)}{M_{P}^{2}} \mathcal{W}\left(p_{0}\right)
$$

whereas local maximum satisfies

$$
\left|\partial_{z}^{2} \mathcal{W}\left(p_{0}\right)\right|<\frac{g_{z \bar{z}}\left(p_{0}\right)}{2 M_{P}^{2}} \mathcal{W}\left(p_{0}\right)
$$

Furthermore saddle point occurs if

$$
\frac{g_{z \bar{z}}\left(p_{0}\right)}{2 M_{P}^{2}} \mathcal{W}\left(p_{0}\right)<\left|\partial_{z}^{2} \mathcal{W}\left(p_{0}\right)\right|<\frac{g_{z \bar{z}}\left(p_{0}\right)}{M_{P}^{2}} \mathcal{W}\left(p_{0}\right)
$$

There is a possibility of having degenerate critical points of $V(z, \bar{z})$, namely,

$$
\begin{aligned}
\left|\partial_{z}^{2} \mathcal{W}\left(p_{0}\right)\right| & =\frac{g_{z \bar{z}}\left(p_{0}\right)}{2 M_{P}^{2}} \mathcal{W}\left(p_{0}\right), \\
\left|\partial_{z}^{2} \mathcal{W}\left(p_{0}\right)\right| & =\frac{g_{z \bar{z}}\left(p_{0}\right)}{M_{P}^{2}} \mathcal{W}\left(p_{0}\right) .
\end{aligned}
$$

Our comments are in order. From equations (3.3)-(3.4) and (3.7)-(3.9) we find that any local minima of $\mathcal{W}(z, \bar{z})$ are mapped into local maxima of the scalar potential $V(z, \bar{z})$. On the other side, saddles of $\mathcal{W}(z, \bar{z})$ are mapped into saddles or local minima of the scalar potential $V(z, \bar{z})$. Moreover the first equation in Eq. (3.10) comes naturally from the fact that degenerate critical points of $\mathcal{W}(z, \bar{z})$, i.e., Eq. (3.5), are mapped into degenerate critical points of $V(z, \bar{z})$. In this case, both Hessian matrix $H_{\mathcal{W}}$ and $H_{\mathcal{V}}$ are singular. These special points are called intrinsic degenerate critical points of $V(z, \bar{z})$. On the other hand, the second equation in Eq. (3.10) means that some saddle points of $\mathcal{W}(z, \bar{z})$ are mapped into some degenerate critical points of $V(z, \bar{z})$. Note that for a shake of consistency we have assumed that $g_{z \bar{z}}\left(p_{0}\right)>0$.

In the model there are two possibilities of Lorentz invariant $N=1$ vacuums related to the critical point $p_{0}$ of $\mathcal{W}(z, \bar{z})^{7}$. First, the critical point describing Minkowskian spacetime satisfies the following conditions

$$
W\left(z_{0}\right)=\frac{d W}{d z}\left(z_{0}\right)=0 .
$$

\footnotetext{
${ }^{7}$ In the following we mention Lorentz invariant $N=1$ vacuums only as vacuums or ground states.
} 
Moreover, it follows that both eigenvalues and determinant in Eqs. (3.1) and (3.6) can be simplified as follows,

$$
\begin{aligned}
\lambda_{1,2}^{\mathcal{W}} & = \pm e^{K\left(p_{0}\right) / 2 M_{P}^{2}}\left|\frac{d^{2} W}{d z^{2}}\left(z_{0}\right)\right| \\
\operatorname{det} H_{\mathcal{W}} & =-e^{K\left(p_{0}\right) / M_{P}^{2}}\left|\frac{d^{2} W}{d z^{2}}\left(z_{0}\right)\right|^{2}, \\
\lambda_{1,2}^{V} & =2 g^{z \bar{z}}\left(p_{0}\right) e^{K\left(p_{0}\right) / M_{P}^{2}}\left|\frac{d^{2} W}{d z^{2}}\left(z_{0}\right)\right|^{2}, \\
\operatorname{det} H_{V} & =4 g_{z \bar{z}}^{-2}\left(p_{0}\right) e^{2 K\left(p_{0}\right) / M_{P}^{2}}\left|\frac{d^{2} W}{d z^{2}}\left(z_{0}\right)\right|^{4} .
\end{aligned}
$$

Non-degeneracy requires

$$
\frac{d^{2} W}{d z^{2}}\left(z_{0}\right) \neq 0
$$

which follows that the superpotential $W(z)$ has to be at least quadratic form. In other words, the family of Minkowskian ground states satisfying Eq. (3.13) is said to be isolated. Moreover, in these vacuums the possible non-degenerate critical points of $\mathcal{W}(z, \bar{z})$ are saddles which are mapped into the minima of the scalar potential, $V(z, \bar{z})$. Both critical points of $\mathcal{W}(z, \bar{z})$ and the scalar potential (2.2) will be degenerate or called non-isolated if Eq. (3.13) vanishes. An interesting feature of this case is that these properties do not change if we set $M_{P} \rightarrow+\infty$. This means that there is an isomorphism between Minkowskian vacuums in local and global supersymmetric theories for $W\left(z_{0}\right)=0$. Finally we want to note that although the first order analysis does not depend on the $U(1)$ connection, however in general if we include the higher order terms it does play a role in Minkowskian vacuums.

On the other hand, the critical point describing Anti-de Sitter (AdS) spacetime has to be obeyed by the following conditions

$$
W\left(z_{0}\right) \neq 0, \quad \nabla_{z} W\left(p_{0}\right) \equiv \frac{d W}{d z}\left(z_{0}\right)+\left(K_{z}\left(z_{0}, \bar{z}_{0}\right) / M_{P}^{2}\right) W\left(z_{0}\right)=0 .
$$

Isolated AdS ground states demand that the function $\mathcal{W}(z, \bar{z})$ must not satisfy Eq. (3.10). Unlike Minkowskian vacuums, the first order analysis of AdS vacuums does depend on the $U(1)$-connection i.e., the term $\left(K_{z}\left(z_{0}, \bar{z}_{0}\right) / M_{P}^{2}\right)$ which is non-holomorphic, beside superpotential $W(z)$. To solve such non-holomorphic equation we choose the critical points of the real function $\mathcal{W}(z, \bar{z})$ are determined by the critical points of the holomorphic superpotential $W(z)$, i.e., that

$$
\frac{d W}{d z}\left(z_{0}\right)=0
$$

which implies that

$$
K_{z}\left(z_{0}, \bar{z}_{0}\right)=K_{\bar{z}}\left(z_{0}, \bar{z}_{0}\right)=0,
$$

since $W\left(z_{0}\right) \neq 0$. This also means that at the ground states the $U(1)$-connection does not appear in any order analysis. This case will be discussed in section 5 . 


\section{Gradient Flows versus RG Flows}

In this section we discuss properties of the gradient flow equations (2.8) and RG flow described by the beta function (2.9) around critical points (or equilibrium points) of $\mathcal{W}(z, \bar{z})$.

Let us first consider the gradient flows using dynamical system analysis [11]. We say that $p_{0}$ is an equilibrium point of the gradient flow equation (2.8) if

$$
\begin{aligned}
& z^{\prime}\left(p_{0}\right)=\mp 2 g^{z \bar{z}} \bar{\partial}_{\bar{z}} \mathcal{W}\left(p_{0}\right)=0, \\
& \bar{z}^{\prime}\left(p_{0}\right)=\mp 2 g^{z \bar{z}} \partial_{z} \mathcal{W}\left(p_{0}\right)=0,
\end{aligned}
$$

are fulfilled. These follow that the equilibrium points of Eq. (2.8) are also the critical points of $\mathcal{W}(z, \bar{z})$. We then expand Eq. (2.8) near its equilibrium point up to first order. It turns out that the first order expansion matrix has eigenvalues

$$
\Lambda_{1,2}=\mp g^{z \bar{z}}\left(p_{0}\right) \lambda_{1,2}^{\mathcal{W}}=\mp \frac{\mathcal{W}\left(p_{0}\right)}{M_{P}^{2}}-2 g^{z \bar{z}}\left(p_{0}\right)\left|\partial_{z}^{2} \mathcal{W}\left(p_{0}\right)\right| .
$$

Stable flow further demands that the two eigenvalues have to be negative due to Lyapunov theorem. We then find

$$
\left|\partial_{z}^{2} \mathcal{W}\left(p_{0}\right)\right|>\frac{g_{z \bar{z}}\left(p_{0}\right)}{2 M_{P}^{2}} \mathcal{W}\left(p_{0}\right)
$$

which are stable nodes. Comparing Eq. (4.3) with Eqs. (3.7) and (3.9) we see that these flows are flowing along local minima and the stable directions of saddles of the scalar potential (2.10). In other words, the evolution of domain walls is stable in the context of dynamical system. For unstable flow in the model we have only unstable saddle since Eq. (4.2) has one possible positive eigenvalue if the following condition

$$
\left|\partial_{z}^{2} \mathcal{W}\left(p_{0}\right)\right|<\frac{g_{z \bar{z}}\left(p_{0}\right)}{2 M_{P}^{2}} \mathcal{W}\left(p_{0}\right)
$$

is satisfied. This condition occurs only at the local maximum of the scalar potential, namely, the condition (3.8). Our linear analysis fails when the Hessian matrix $H_{\mathcal{W}}$ becomes singular. As discussed in previous section, this occurs at intrinsic degenerate critical points of the scalar potential $V(z, \bar{z})$. In other words, the condition (3.5) is fulfilled. In dynamical system picture these points could be a bifurcation point of the gradient flow equation (2.8). Since we have only a zero eigenvalue, then such bifurcation is called fold bifurcation [11]. These points are assured by at least one of the higher order terms evaluated at the ground states in Taylor expansion of Eq. (2.8), namely,

$$
\begin{aligned}
\partial_{z}^{n}\left(g^{z \bar{z}} \partial_{z} \mathcal{W}\right)\left(p_{0}\right) & =\sum_{q=0}^{n} \frac{n !}{(n-q) !} \partial_{z}^{q} g^{z \bar{z}}\left(p_{0}\right) \partial_{z}^{n-q+1} \mathcal{W}\left(p_{0}\right) \\
\partial_{\bar{z}}^{m}\left(g^{z \bar{z}} \partial_{z} \mathcal{W}\right)\left(p_{0}\right) & =\sum_{q=0}^{m} \frac{m !}{(m-q) !} \partial_{\bar{z}}^{q} g^{z \bar{z}}\left(p_{0}\right) \partial_{\bar{z}}^{m-q} \partial_{z} \mathcal{W}\left(p_{0}\right)
\end{aligned}
$$

for $m \geq 2, n \geq 2$,

$$
\partial_{\bar{z}}^{m} \partial_{z}^{n}\left(g^{z \bar{z}} \partial_{z} \mathcal{W}\right)\left(p_{0}\right)=\sum_{q=0}^{n} \sum_{r=0}^{m} \frac{n !}{(n-q) !} \frac{m !}{(m-r) !} \partial_{\bar{z}}^{r} \partial_{z}^{q} g^{z \bar{z}}\left(p_{0}\right) \partial_{\bar{z}}^{m-q} \partial_{z}^{n-q+1} \mathcal{W}\left(p_{0}\right)
$$


for $m \geq 1, n \geq 1$, and their complex conjugate is have to be non-zero.

As discussed in the previous section, in Minkowskian vacuums the eigenvalue of Eq. (4.2) can be negative or zero. For negative value, the flows are guaranteed to be stable flowing around isolated minimal Minkowskian ground states, while our linear analysis failed when it vanishes. In AdS cases, there are two possible flows, namely, stable nodes or unstable saddles.

Now we turn to consider the RG flows. As we have mentioned in section 2, this function can also be used to determine the nature of the critical point $p_{0}$, namely, it can be interpreted as UV or IR in the CFT picture. To begin, we expand the beta function (2.9) to first order around $p_{0}$ and then we have the matrix

$$
\mathcal{U} \equiv-\left(\begin{array}{cc}
\frac{\partial \beta}{\partial z}\left(p_{0}\right) & \frac{\partial \bar{\beta}}{\partial z}\left(p_{0}\right) \\
\frac{\partial \beta}{\partial \bar{z}}\left(p_{0}\right) & \frac{\partial \bar{\beta}}{\partial \bar{z}}\left(p_{0}\right)
\end{array}\right)
$$

whose eigenvalues are

$$
\lambda_{1,2}^{\mathcal{U}}=\frac{2 g^{z \bar{z}}\left(p_{0}\right)}{\mathcal{W}\left(p_{0}\right)} \lambda_{1,2}^{\mathcal{W}}=\frac{2}{M_{P}^{2}} \pm \frac{4 g^{z \bar{z}}\left(p_{0}\right)}{\mathcal{W}\left(p_{0}\right)}\left|\partial_{z}^{2} \mathcal{W}\left(p_{0}\right)\right|
$$

Let us choose a model where the UV region is $u \rightarrow+\infty$ as $a \rightarrow+\infty$, while the IR region is $u \rightarrow-\infty$ as $a \rightarrow 0$. First we consider the UV critical points. In UV region at least one of the eigenvalues (4.8) should be positive. Since it is possible to have zero and a negative eigenvalue as the parameters in $\mathcal{W}(z, \bar{z})$ vary, namely, in the direction of

$$
\lambda_{2}^{\mathcal{U}}=\frac{2}{M_{P}^{2}}-\frac{4 g^{z \bar{z}}\left(p_{0}\right)}{\mathcal{W}\left(p_{0}\right)}\left|\partial_{z}^{2} \mathcal{W}\left(p_{0}\right)\right|,
$$

the $\mathrm{RG}$ flows fail to depart the UV region in this direction. Thus, this flow is stable along

$$
\lambda_{1}^{\mathcal{U}}=\frac{2}{M_{P}^{2}}+\frac{4 g^{z \bar{z}}\left(p_{0}\right)}{\mathcal{W}\left(p_{0}\right)}\left|\partial_{z}^{2} \mathcal{W}\left(p_{0}\right)\right|
$$

Correspondingly, the local maxima of the scalar potential $V(z, \bar{z})$ are changing into saddles or local minima in this UV region. Then the stability of gradient flows is changing, namely, from unstable saddles into stable nodes. In addition, it is easy to see that bifurcation point of gradient flows does exist in UV region. On the other hand, in IR region the RG flow approaches a critical point in the direction where the eigenvalue of Eq. (4.9) is negative. However, it is failed when Eq. (4.9) vanishes and then becomes positive. Thus, IR critical points vanish as the parameters varying. In other words, there is no IR local maximum of the scalar potential and the gradient flows do not exist around this point in the model. Moreover, we have no bifurcation point of gradient flows in this region.

\section{Models}

We organize this section into three parts. First we consider a model where the superpotential are algebraic polynomials, namely, linear, quadratic, and cubic polynomials. Then in the second part a model with harmonic superpotential will be discussed. The last part 
will be a model with elliptic superpotential which describes Riemann surfaces of genus one. For the rest of the paper we take the Kähler potential to be

$$
K(z, \bar{z})=\left|z-z_{0}\right|^{2}
$$

where the parameter $z_{0}$ is chosen such that in vacuums it satisfies Eq. (3.16). Then this choice further simplifies Eqs. (3.1) and (3.6) into

$$
\begin{aligned}
\lambda_{1,2}^{\mathcal{W}} & =\frac{\left|W\left(z_{0}\right)\right|}{M_{P}^{2}} \pm\left|\frac{d^{2} W}{d z^{2}}\left(z_{0}\right)\right| \\
\operatorname{det} H_{\mathcal{W}} & =\frac{\left|W\left(z_{0}\right)\right|^{2}}{M_{P}^{4}}-\left|\frac{d^{2} W}{d z^{2}}\left(z_{0}\right)\right|^{2}, \\
\lambda_{1,2}^{V} & =2\left(\left|\frac{d^{2} W}{d z^{2}}\left(z_{0}\right)\right|^{2}-\frac{2\left|W\left(z_{0}\right)\right|^{2}}{M_{P}^{4}} \pm \frac{\left|W\left(z_{0}\right)\right|}{M_{P}^{2}}\left|\frac{d^{2} W}{d z^{2}}\left(z_{0}\right)\right|\right), \\
\operatorname{det} H_{V} & =4\left(\frac{\left|W\left(z_{0}\right)\right|^{2}}{M_{P}^{4}}-\left|\frac{d^{2} W}{d z^{2}}\left(z_{0}\right)\right|^{2}\right)\left(\frac{4\left|W\left(z_{0}\right)\right|^{2}}{M_{P}^{4}}-\left|\frac{d^{2} W}{d z^{2}}\left(z_{0}\right)\right|^{2}\right) .
\end{aligned}
$$

We see that in this model the dynamics of the walls are completely determined by the superpotential $W(z)$. As mentioned in section 3 Minkowskian vacuums are stable and isolated for at least quadratic form in both local and global $N=1$ supersymmetric theories.

\subsection{A Model with $W(z)$ as Algebraic Polynomials}

In this section we consider a model in which the holomorphic superpotential has the following form

$$
W(z)=\sum_{n=0}^{\mathcal{N}} a_{n} z^{n},
$$

with $a_{n}, n=0, \ldots, \mathcal{N}$ are complex. In particular we will focus, as examples, on $\mathcal{N}=0,1,2$.

Let us first consider two simplest models, namely, $\mathcal{N}=0$ and linear model $\mathcal{N}=1$. In these models we have

$$
\begin{aligned}
& \lambda_{1,2}^{\mathcal{W}}=\frac{\left|a_{0}\right|}{M_{P}^{2}}, \quad \Lambda_{1,2}=\mp \frac{\left|a_{0}\right|}{M_{P}^{2}} \\
& \lambda_{1,2}^{V}=-4 \frac{\left|a_{0}\right|^{2}}{M_{P}^{4}}, \quad V\left(z_{0}, \bar{z}_{0}\right)=-\frac{3\left|a_{0}\right|^{2}}{M_{P}^{2}}
\end{aligned}
$$

since the critical points are trivial for $\mathcal{N}=0$ and $a_{1}=0$ for $\mathcal{N}=1$. AdS vacuums occur if $a_{0} \neq 0$ which are unstable and isolated, while these models admit trivial Minkowskian vacuums if $a_{0}=0$ which is not a bifurcation point. Furthermore, in both models we have only UV vacuums. Note that these two models are degenerate in the sense that they have the same properties at the ground states. 
Now we turn to consider the case for $\mathcal{N}=2$. In the model we have

$$
\begin{aligned}
\lambda_{1,2}^{\mathcal{W}} & =\frac{|D|}{4\left|a_{2}\right| M_{P}^{2}} \pm 4\left|a_{2}\right|, \\
\Lambda_{1,2} & =\mp \frac{|D|}{4\left|a_{2}\right| M_{P}^{2}}-4\left|a_{2}\right| \\
\lambda_{1,2}^{V} & =8\left|a_{2}\right|^{2}-\frac{|D|^{2}}{16\left|a_{2}\right|^{2} M_{P}^{4}} \pm \frac{|D|}{2 M_{P}^{2}}, \\
V\left(z_{0}, \bar{z}_{0}\right) & =-\frac{3|D|^{2}}{16\left|a_{2}\right|^{2} M_{P}^{2}}
\end{aligned}
$$

where $D=a_{1}^{2}-4 a_{2} a_{0}$. Clearly, Minkowskian vacuums emerge if $D=0$ and $a_{2} \neq 0$ which are stable and isolated in UV or IR regions. The case where $D=0$ and $a_{2}=0$ one should regain the previous models which are AdS for $a_{0} \neq 0$. Particularly, the analysis is blown up for $D \neq 0$ and $a_{2}=0$ in UV region.

\subsection{A Model with Harmonic $W(z)$}

This section is devoted to discuss properties of a model where the superpotential $W(z)$ has the harmonic form ${ }^{8}$

$$
W(z)=A_{0} \sin (k z)
$$

where $A_{0}$ and $k$ are real. Furthermore, by defining $z \equiv x+\mathrm{i} y$, the critical points are

$$
y=0, x=\left(n+\frac{1}{2}\right) \frac{\pi}{k}, \quad n=0, \pm 1, \pm 2, \ldots
$$

Then the eigenvalues in Eqs. (5.2) and (3.1) and the scalar potential (2.10) becomes

$$
\begin{aligned}
\lambda_{1,2}^{\mathcal{W}} & =\left(\frac{1}{M_{P}^{2}} \pm k^{2}\right)\left|A_{0}\right| \\
\Lambda_{1,2} & =\left(\mp \frac{1}{M_{P}^{2}}-k^{2}\right)\left|A_{0}\right| \\
\lambda_{1,2}^{V} & =2\left|A_{0}\right|^{2}\left(k^{4}-\frac{2}{M_{P}^{4}} \pm \frac{k^{2}}{M_{P}^{2}}\right), \\
V\left(z_{0}, \bar{z}_{0}\right) & =-\frac{3\left|A_{0}\right|^{2}}{M_{P}^{2}} .
\end{aligned}
$$

In this model we have only nontrivial AdS vacuums and the possible bifurcation point happens at $k= \pm \frac{1}{M_{P}}$.

\subsection{A Model with Elliptic $W(z)$}

In this section we give example of a model where the holomorphic superpotential $W(z)$ can be viewed as Weierstrass function, [13] ${ }^{9}$

$$
\left(\frac{d W(z ; \tau)}{d z}\right)^{2}=\left(W(z ; \tau)-e_{1}(\tau)\right)\left(W(z ; \tau)-e_{2}(\tau)\right)\left(W(z ; \tau)-e_{3}(\tau)\right)
$$

\footnotetext{
${ }^{8}$ This harmonic form of superpotential with gravitational correction has been discussed in Ref. [12].

${ }^{9}$ This function has been studied in the context of dilaton domain walls (Ref. [14]).
} 
where the roots $e_{1}, e_{2}, e_{3}$ are complex and moduli $\tau$-dependent. These can be expressed in terms of theta functions $\vartheta(0 ; \tau)$,

$$
\begin{aligned}
& e_{1}(\tau)=\frac{\pi^{2}}{3}\left(\vartheta^{4}(0 ; \tau)+\vartheta_{01}^{4}(0 ; \tau)\right) \\
& e_{2}(\tau)=-\frac{\pi^{2}}{3}\left(\vartheta^{4}(0 ; \tau)+\vartheta_{10}^{4}(0 ; \tau)\right), \\
& e_{3}(\tau)=-\left(e_{1}(\tau)+e_{2}(\tau)\right)
\end{aligned}
$$

where

$$
\begin{aligned}
\vartheta(z ; \tau) & =\sum_{n=-\infty}^{+\infty} q^{n^{2} / 2} e^{2 \pi \mathrm{i} n z} \\
\vartheta_{01}(z ; \tau) & =\sum_{n=-\infty}^{+\infty}(-1)^{n} q^{n^{2} / 2} e^{2 \pi \mathrm{i} n z}, \\
\vartheta_{10}(z ; \tau) & =\sum_{n=-\infty}^{+\infty} q^{\frac{1}{2}\left(n+\frac{1}{2}\right)^{2}} e^{(2 n+1) \pi \mathrm{i} z}
\end{aligned}
$$

with $q \equiv e^{2 \pi \mathrm{i} \tau}$. Furthermore the superpotential $W(z ; \tau)$ satisfying Eq. (5.9) can also be written down in terms of theta functions $\vartheta(z ; \tau)$,

$$
W(z ; \tau)=\pi^{2} \vartheta^{2}(0 ; \tau) \vartheta_{10}^{2}(0 ; \tau) \frac{\vartheta_{01}^{2}(z ; \tau)}{\vartheta_{11}^{2}(z ; \tau)}+e_{2}(\tau)
$$

where

$$
\vartheta_{11}(z ; \tau)=\sum_{n=-\infty}^{+\infty}(-1)^{n} q^{\frac{1}{2}\left(n+\frac{1}{2}\right)^{2}} e^{(2 n+1) \pi \mathrm{i} z} .
$$

Now we recall about critical points of $\mathcal{W}(z, \bar{z})$. For the case at hand we find that if $p_{0}$ is a critical point of $\mathcal{W}(z, \bar{z})$, then we have

$$
W\left(z_{0} ; \tau\right)=e_{l}(\tau), \quad l=1,2,3 .
$$

Then the scalar potential (2.2) becomes

$$
V(\tau, \bar{\tau})=-\frac{3\left|e_{l}(\tau)\right|^{2}}{M_{P}^{2}} \quad, \text { (no summation) }
$$

which is the cosmological constant of the model. However our analysis (5.2) becomes ill defined since it diverges at $p_{0}$. These singularities cannot be removed and can be found in another part of the upper-half plane by employing modular transformation with respect to $\tau$. This means that our analysis fails for this function near its critical points.

\section{Conclusions}

In this paper we have studied general properties of $N=1$ vacuums of BPS equations and the scalar potential in four dimensional $N=1$ supergravity coupled to a chiral multiplet. 
Firstly, our analysis on the real function $\mathcal{W}(z, \bar{z})$ shows that this function admits three types of critical points, namely, local minima, saddles, and degenerate critical points. The local minima are mapped into local maxima of the scalar potential, while the saddles are mapped into local minima or saddles of the scalar potential. Moreover, degeneracy of $\mathcal{W}(z, \bar{z})$ also implies degeneracy of the scalar potential. These points are called intrinsic degenerate critical points of the scalar potential.

Secondly, using dynamical system analysis on gradient flow equations we obtain that in this model these flows are unstable near local maximum of the scalar potential, whereas they are stable near saddles, local minimum, and degenerate critical points of the scalar potential. Furthermore, we have showed that in this model it is possible to have a bifurcation point occurring near intrinsic degenerate critical points of the scalar potential. Thirdly, to check the existence of such flows near critical points one has to perform the analysis on the beta function. Our conclusion is that IR local maxima does not exist in the model which further tells us that no gradient flows exist around these points in the region.

Finally, we have considered three models where the ground states are completely controlled by the superpotential. In the model with algebraic polynomials we find that there is degeneracy between the two models, namely, constant and linear superpotentials. These simple models admit nontrivial unstable and isolated AdS vacuums in UV region. For the case of quadratic polynomial the situation is more complicated. In particular, our analysis diverges for $D \neq 0$ and $a_{2}=0$ in UV region.

In the model with harmonic superpotential we have periodic critical points and then the nontrivial ground states are AdS. Lastly, the model with elliptic superpotential has also been considered. However, our analysis becomes divergence near critical points in this model.

\section{A Convention and Notation}

The purpose of this appendix is to collect our conventions in this paper. The spacetime metric is taken to have the signature $(+,-,-,-)$ while the Ricci scalar is defined to be

$R=g^{\mu \nu}\left[\frac{1}{2} \partial_{\rho} \Gamma_{\mu \nu}^{\rho}-\partial_{\nu} \Gamma_{\mu \rho}^{\rho}+\Gamma_{\mu \nu}^{\sigma} \Gamma_{\sigma \rho}^{\rho}\right]+\frac{1}{2} \partial_{\rho}\left[g^{\mu \nu} \Gamma_{\mu \nu}^{\rho}\right]$. The Christoffel symbol is given by $\Gamma_{\nu \rho}^{\mu}=\frac{1}{2} g^{\mu \sigma}\left(\partial_{\nu} g_{\rho \sigma}+\partial_{\rho} g_{\nu \sigma}-\partial_{\sigma} g_{\nu \rho}\right)$ where $g_{\mu \nu}$ is the spacetime metric.

The following indices are given: 


$$
\begin{aligned}
\bar{\mu}, \bar{\nu}=0,1,2, & \text { label curved three dimensional spacetime indices } \\
\bar{a}, \bar{b}=0,1,2, & \text { label flat three dimensional spacetime indices } \\
\mu, \nu=0, \ldots, 3, & \text { label curved four dimensional spacetime indices } \\
a, b=0, \ldots, 3, & \text { label flat four dimensional spacetime indices }
\end{aligned}
$$

\section{B Hessian Matrix}

Let us first consider an arbitrary (real) $C^{\infty}$-function $f(z, \bar{z})$. A point $p_{0}=\left(z_{0}, \bar{z}_{0}\right)$ is said to be a critical point of $f(z, \bar{z})$ if the following conditions

$$
\frac{\partial f}{\partial z}\left(p_{0}\right)=0, \quad \frac{\partial f}{\partial \bar{z}}\left(p_{0}\right)=0,
$$

hold. Furthermore, the point $p_{0}$ is a non-degenerate critical point of $f(z, \bar{z})$ if the Hessian matrix

$$
H_{f} \equiv 2\left(\begin{array}{cc}
\frac{\partial^{2} f}{\partial z \partial \bar{z}}\left(p_{0}\right) & \frac{\partial^{2} f}{\partial z^{2}}\left(p_{0}\right) \\
\frac{\partial^{2} f}{\partial \bar{z}^{2}}\left(p_{0}\right) & \frac{\partial^{2} f}{\partial \bar{z} \partial z}\left(p_{0}\right)
\end{array}\right)
$$

is non-singular, i.e.,

$$
\operatorname{det} H_{f}=4\left[\left(\frac{\partial^{2} f}{\partial z \partial \bar{z}}\left(p_{0}\right)\right)^{2}-\frac{\partial^{2} f}{\partial z^{2}}\left(p_{0}\right) \frac{\partial^{2} f}{\partial \bar{z}^{2}}\left(p_{0}\right)\right] \neq 0 .
$$

The eigenvalues of the Hessian matrix $H_{f}$ are given by

$$
\begin{aligned}
\lambda_{1}^{f} & =\frac{1}{2}\left(\operatorname{tr} H_{f}+\sqrt{\left(\operatorname{tr} H_{f}\right)^{2}-4 \operatorname{det} H_{f}}\right), \\
\lambda_{2}^{f} & =\frac{1}{2}\left(\operatorname{tr} H_{f}-\sqrt{\left(\operatorname{tr} H_{f}\right)^{2}-4 \operatorname{det} H_{f}}\right) .
\end{aligned}
$$

Now we can characterize the critical point $p_{0}$ of the function $f$ using the eigenvalues defined in Eq. (B.4) as follows:

1. If two eigenvalues are positive, i.e., $\lambda_{1}^{f}>0$ and $\lambda_{2}^{f}>0$, then $p_{0}$ is a local minimum.

This implies

$$
\begin{aligned}
\operatorname{tr} H_{f} & >0, \\
\operatorname{det} H_{f} & >0 .
\end{aligned}
$$


2. If two eigenvalues are negative, i.e., $\lambda_{1}^{f}<0$ and $\lambda_{2}^{f}<0$, then $p_{0}$ is a local maximum which satisfies

$$
\begin{aligned}
\operatorname{tr} H_{f} & <0, \\
\operatorname{det} H_{f} & >0 .
\end{aligned}
$$

3. If we have $\lambda_{1}^{f}>0$ and $\lambda_{2}^{f}<0$ or vice versa, then $p_{0}$ is a saddle point which satisfies

$$
\operatorname{det} H_{f}<0 \text {. }
$$

4. Finally if at least one eigenvalue vanishes, then $p_{0}$ is a degenerate critical point. This means that we have

$$
\operatorname{det} H_{f}=0
$$




\section{Acknowledgement}

We acknowledge H. Alatas and J.M. Tuwankotta for nice discussion about bifurcation theory. We would like to thank M. Cvetic for notifying Refs. [3] and [4] and K. Yamamoto for informing us Ref. [6]. This work is supported by Riset ITB 2005 No. 0004/K01.03.2/PL2.1.5/I/2006.

\section{References}

[1] For a review see, for example:

O. Aharony, S. S. Gubser, J. Maldacena, H. Ooguri, and Y. Oz, Phys. Rep. 323183 (2000), and references therein, e-print:hep-th/9905111.

[2] A. Celi, Ph.D. Thesis Universita d'Milano, and references therein, e-print:hepth/0405283.

[3] M. Cvetic, S. Griffies and S.J. Rey, Nucl. Phys. B 381301 (1992), e-print:hepth/9201007.

[4] M. Cvetic and H.H. Soleng, Phys. Rep. 282159 (1997), e-print:hep-th/9604090.

[5] J. Sonner and P.K. Townsend, Class. Quantum Grav. 23, 441 (2006), e-print:hepth/0510115.

[6] A. Ceresole, G. Dall'Agata, A. Girvayets, R. Kallosh, and A. Linde, Domain walls, near-BPS bubbles and probabilities in the landscape, Phys. Rev. D74 (2006) 086010, hep-th/0605266.

[7] J. Wess and J. Bagger, Supersymmetry and Supergravity, 2nd ed. ( Princeton University Press, Princeton 1992). R. D'Auria and S. Ferrara , JHEP 0105, 034 (2001), e-print:hep-th/0103153. L. Andrianopoli, R. D'Auria, and S. Ferrara, JHEP 0203, 025 (2002), e-print:hep-th/0110277.

[8] D.Z. Freedman, S.S. Gubser, K. Pilch and N.P. Warner, Renormalization group flows from holography-supersymmetry and a c-theorem, Adv. Theor. Math. Phys. 3 (1999) 363, hep-th/9904017.

[9] A. Ceresole, G. Dall'Agata, R. Kallosh, and A. van Proeyen, Hypermultiplets, Domain Walls and Supersymmetric Attractor, Phys. Rev. D64 (2001) 104006, hepth/0104056.

[10] W. Kaplan, Advanced Calculus, (2 ed.), Addison Wesley (1973).

[11] Y. A. Kuznetsov, Elements of Applied Bifurcation Theory, Springer-Verlag New York (1998).

[12] M. Eto, N. Maru, N. Sakai, and T. Sakata, Exactly solved BPS wall and winding number in $N=1$ Supergravity, Phys. Lett. B553 (2003) 87, hep-th/0208127. 
[13] H. M. Farkas and I. Kra, Riemann Surfaces, Springer-Verlag New York (1980). N. Koblitz, Introduction to Elliptic Curves and Modular Forms, Springer-Verlag New York (1993).

[14] K. Skenderis and P.K. Townsend, Gravitational Stability and Renormalization Group Flow, Phys. Lett. B468 (1999) 46, hep-th/9909070. 